\title{
A Proactive Energy-Awareness Based Traffic Routing in Tree Topology Wireless Sensor Networks Precluding Energy Holes Formation
}

\author{
Mayada S. A. Mustafa, Borhanuddin M. Ali *, Mohd F. A Rasid and Shaiful J. B. Hashim \\ 1 Department of Computer and Communication Systems Engineering, Wireless and Photonics Research \\ Centre (WiPNET), Faculty of Engineering, Universiti Putra Malaysia, 43400 UPM Serdang, Selangor, \\ Malaysia; E-Mails ; m_ysra@yahoo.com \{borhan, fadlee, sjh\}@upm.edu.my \\ * Correspondence: borhan@upm.edu.my
}

\begin{abstract}
A single tree topology is a commonly employed topology for wireless sensor networks (WSN) to connect sensors to one or more remote gateways. However, its many-to-one traffic routing pattern imposes heavy burden on downstream nodes, as the same routes are repeatedly used for packet transfer, from one or more upstream branches. The challenge is how to choose the most optimal routing paths that minimizes energy consumption across the entire network. This paper proposes a proactive energy awareness-based many-to-one traffic routing scheme to alleviate the above said problem referred to as Energy Balance-Based Energy Hole Alleviation in tree topology (EBEHA-T). This protocol combines updated status of variations in energy consumption pattern around sink-hole zones and distribution of joint nodes among the trees. With this approach, EBEHA$\mathrm{T}$ proactively prevents sink-hole formation instead of just a reactive response after they have occurred. Performance evaluation of EBEHA-T against benchmark method RaSMaLai shows increased energy saving across the entire network and a marked improvement in energy consumption balance in energy-hole zones. This precludes energy hole formation and the consequent network partitioning, leading to improved network lifetime beyond that of the RasMaLai. OMNET++ network simulation software has been used for the evaluation.
\end{abstract}

Keywords: WSN; lifetime; energy hole; tree topology; routing.

\section{Introduction}

Wireless sensor nodes are energy constrained devices which can transfer sensed data from the field to servers in remote locations. This are generally done over multi-hop networks comprising of other similar nodes. Sensor nodes that are one-hop away from their respective gateways (referred to as deputy nodes, DNs) are responsible for major traffic delivery to this node, hereinafter referred to as the sink node. In the evaluation of suitable wireless sensor networks (WSN) topology for network lifetime, the first sensor node that runs out of energy constitutes one important metric [1]. Fast energy depletion at DNs creates energy holes that would consequently cause the isolation of sink nodes and henceforth, short network lifetime [2,3]. Because of the sensor nodes energy constraint, the issue of energy consumption in the network has become the subject of much research on how to prolong the lifetime of the network [4,5]. In recent years, balanced energy consumption over the network has been identified as one of the promising solutions for optimal energy consumption [6-8].

Several studies have addressed the problem of energy consumption from different perspectives [9-12]. In [13] the authors proposed cooperative routing to minimize energy consumption at critical 
zones. From the energy information of the nodes, the approach adapts the communication range of the sensor nodes transmission based on the signal-to-noise ratio of the channel.

The authors in [14] proposed a mobile sink approach to a tree-cluster based data-gathering algorithm (TCBDGA) that balances the load of the entire network. The mobile sink avoids locations of heavily loaded zones by re-selecting its rendezvous point (RPs) for data collection. The study in [15] proposed dividing the network into hierarchical circles where the inner ones decrease gradually in size as they get closer to the sink nodes. Each layer is divided into sectors each with a header node to accumulate data. Such topology management reduces data traffic and select node efficiently to forward the data. In [16] energy-aware balanced energy consumption and hole alleviating algorithm (BECHA) was proposed with an optimal forwarding strategy to achieve balanced load distribution among nodes with minimum and maximum energy depletion. The study in [17] presented On-Hole Children Reconnection (OHCR) and On-Hole Alert (OHA) in tree-based topology in which the number of child nodes is addressed as a factor influencing the energy consumption pattern in the network. In [18], load management is proposed to alleviate the hole problem. The cluster heads (CHs) send their data directly to the sink or through "associative headers" based on a distance threshold. An associative header is a cluster member with the highest energy and it will replace the cluster head when an energy hole is detected. Further focus of research goes on determining the most significant parameters in the tree topology that affect energy behavior pattern, particularly at critical zones.

In this paper, we propose a method that performs proactive management of energy consumption, particularly at energy hole zones, which has not been considered in previous researches. Throughout the operation of the network, the energy behavior of distant source nodes may have changed and traffic routings further downstream may have higher capacity. Accordingly, in this paper we propose Energy Balance-Based Energy Hole Alleviation in Tree topology (EBEHA-T) routing mechanism to address this gap. Extensive simulations using Omnet++ have been carried out to evaluate the performance of EBEHA-T. The rest of this paper is organized as follows: Previous works and taxonomy of energy hole remedy in WSN is summarized in Section 2. Node association manners to tree topology and its impact on energy consumption pattern in critical zones is analyzed in Section 3. Description of our proposed work and discussion of simulation results are presented in Section 4 and 5, respectively. Finally, conclusion and future direction of the research are given in Section 6.

\section{Previous works and taxonomy on energy hole remedy}

This section discusses previous works for energy hole in WSN and presents a taxonomy of major remedies as shown in Figure 1 and Figure 2, respectively. The major categories are topology control, node deployment, traffic routing, and hybrid perspectives.

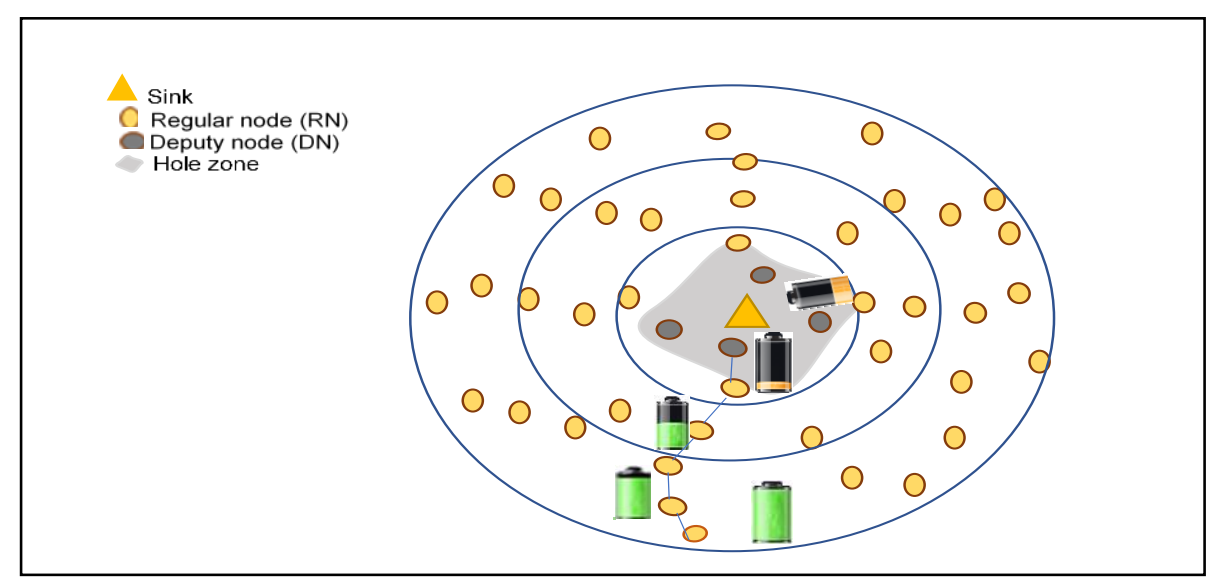

Figure 1. Energy consumption with respect to distance from the sink as indicated by the content of the batteries. 


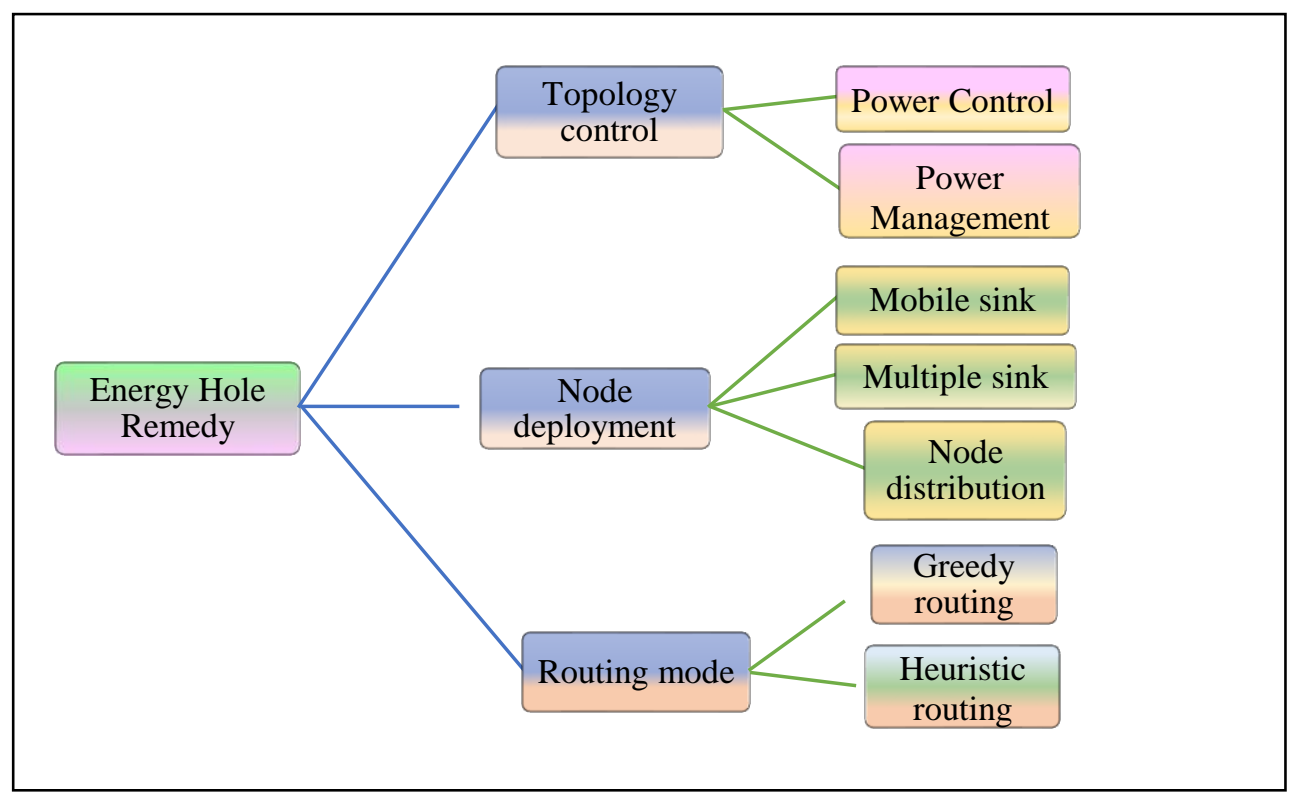

Figure 2. Taxonomy of sink energy hole remedy.

\subsection{Topology control}

Topology control is classified into power control and power management. The following subsections reviews a number of proposals.

\subsubsection{Power control}

Dynamic re-adjustment of transmission power by sensor nodes affect neighbor nodes association, and subsequently network connectivity and coverage. The study in [19] analyzed energy hole problem in multi-hop flat sensor networks, particularly their node locations, and corresponding maximum lifetime. It highlights that energy consumption is related to distance of sensor nodes to the sink node, so it proposes managing transmission range for deployed sensor nodes in a circular area. The idea is that, an adjustable transmit power can optimize transmission energy cost and henceforth, the network lifetime.

The research in [20] proposed transmission power adjustment known as Multihop/Direct Forwarding (MDF). A source node splits its outgoing data into two, either being transmitted directly to the sink or through multi-hop path where each hop has a transmission range of " $\mathrm{h}$ " to reach the destination, to control the overall energy consumption. Direct Forwarding aims to balance energy consumption of nodes at different locations, particularly around energy hole zones. The paper in [21] proposed a transmission range list for improved corona topology model with multiple levels, where each corona level is assigned different transmission range. Two algorithms, Centralized EnergyEfficient Transmission Trees (CETT) and Distributed Energy-Efficient Transmission range List, (DETL) are deployed using transmission lists for uniform and non-uniform node distribution, respectively.

The study in [22] highlighted that optimization of node's transmission radius can balance energy consumption and maximize network lifetime as the number of nodes guarantees coverage and connectivity. The algorithm considers the ratio between network residual energy against a number of transmission ranges to find the optimized transmission radius. The authors in [23] proposed ResidualEnergy-Activated Cooperative Transmission (REACT), where cooperative transmission (CT) is used to avoid burdening nodes around sink nodes and deliver data directly to the destination. REACT triggers CT when the residual energy of next node is lower than that of current node, resulting in a reduction of energy consumption among burdened nodes. 


\subsubsection{Power Management}

Power management is implemented by way of adapting sleep/active state in radio subsystem of sensor nodes [24]. The study in [25] proposed an Energy-efficient Hole Removing Mechanism (EHORM) aimed at energy holes. Each node considers an energy threshold to decide whether to be Active or transit to Sleep state. They turn their transmitter On only during data sending to save some energy. The probability of transition to Sleep state increases as sensor nodes are positioned away from the sink. This way, E-HORM increases network lifetime and stability period. The authors in [26] proposed Carrier Sense Multiple Access with Shortest First (CSMA/SF) for addressing energy hole problems. CSMA/SF is a priority based medium access control instead of contention based one as the name may suggest. Nodes with shorter data are given priority to finish their transmission to avoid continuous sensing of medium, hence reducing energy. Length Detection mechanism cooperatively helps in detecting which are the nodes that have short data to be assigned priority of sending the data packets. Anti-Starvation mechanism is also employed as a timeout mechanism for a node that has not transmitted for a long time, as such the protocol assigns highest priority for such a node to be able to transmit first. Thus, the overall energy consumption is reduced and channel utilization is improved. The paper in [27] proposed Synchronization of Node in Adjacent Annuluses (SNAA) for circular networks. The optimal parent node selection and sleep/wakeup schedule by SNAA aims to achieve energy saving in nodes between adjacent annuluses. The decision to go for Sleep mode is based on the measured ratio between the amount of residual energy at the end of transmission period to the residual energy after T time.

\subsection{Node Deployment}

Many researchers addressed the energy situation in critical zones and proposed different strategies of node deployment. These strategies can be divided into three categories;

\subsubsection{Node distribution}

The authors in [28] presented a non-uniform sensor distribution approach in hole zones called zeta distribution (Zipf) under various parametric distribution in circular areas. Zipf aims at optimizing the density for efficient energy saving regardless of routing protocol that the topology is laid upon. Large number of nodes deployed close to the sink leads to increase in energy saving. The study in [29] proposed a non-uniform deterministic node distribution with gradual increase towards the sink node. Compared to other studies, this strategy satisfies topology coverage and load balancing with fewer number of nodes. The idea of managing data originator nodes and router nodes with respect to virtual coronas is to keep network traffic low. The research in [30] proposes a gradual non-uniform node distribution with respect to traffic density. A set of dormant nodes is identified using a designed formula and deployed near the sink node. When a node energy drops to its energy threshold, immediately its nearby dormant node receives an invitation to become active. Synchronously, the sink node that receives signal for next routing tree construction considers association of the dormant nodes. The authors in [31] proposed load-similar node distribution strategy combined with the Minimum Overlapping Layers (MOL) scheme. The number of nodes increases at zones of higher load. The increase in node distribution is regulated using a common ratio in adjacent coronas. The router node with a higher energy is selected to forwards data traffic to the sink node.

\subsubsection{Mobile sink}

The sink can also be made to move in order to find minimum energy to transmit data to destination. The study in [32] addressed strategies of sink mobility against static sink to overcome inefficiency in energy consumption in hot zones. It proposes radius range of mobility trajectory for the sink node with appropriate transmission duty cycle that results higher energy efficiency. In [33] the authors proposed a distributed tree-based data dissemination (TEDD) scheme integrated with a mobile sink. 
The mobile sink selects neighbor nodes with higher energy to serve as aggregator nodes. The proposed protocol comprises of a number of phases; neighbor discovery, tree construction, gateway selection, and data dissemination. The authors in [34] addressed the necessity to locate anchor nodes that the mobile sink should track. It proposes an improved Ant Colony Optimization (ACO) that aids mobile sink to find an optimal moving trajectory. Distance heuristic factor based ACO aid mobile sinks to communicate with cluster heads $(\mathrm{CH})$ using short-range communications. In [35] a number of rendezvous points (RP) based mobile sink are selected using either energy density based trajectory (EDT) or delay aware energy density based trajectory (DAEDT). EDT considers energy density to select a set of RP and deploy traveling sales person algorithm (TSP) to compute the trajectory. DAEDT employs Quicksort algorithm to find a set of regular nodes (RN) and subsequently use TSP to compute the trajectory. Both mechanisms show increase in energy balance. The research in [36] proposed Mobile Sink-Based Adaptive Immune Energy-Efficient Clustering Protocol (MSIEEP). It uses Adaptive Immune Algorithm (AIA) to find sojourn locations of the mobile sink and the optimum number of CHs and their locations. This minimizes energy consumption in communication process and overhead control packets.

\subsubsection{Multiple sinks}

The aim of multiple sinks deployment whether they are static or mobile is to minimize transmission delay, reduce energy consumption, and subsequently extend the network life time. The authors in [37] proposed Lifetime Optimization Algorithm with Multiple Mobile Sink Nodes for Wireless Sensor Networks (LOA_MMSN) to solve the problem of unbalanced energy consumption and formation of energy hole. The sensor coverage area is divided into grids and anchor nodes are positioned at the center. Each mobile sink moves among these centers and aggregate data in the k-hop range before moving back to the initial anchor node. All sensors are allowed to send data in the vicinity of the sink node. The mobility speed is low and time of data gathering is short. The study in [38] proposed a multi-sink and load-balance routing algorithm (MSLBR) that tackles load balancing of nodes close to the sink node. Same source node is allowed to select different neighbor nodes to forward its data to the sink node. Selection for forwarding nodes is based on its residual energy and short hop count to the sink node. MSLBR proposed round-robin way to select one of sink's neighbor each time traffic is generated. The research in [39] proposed Integer Linear Programming (ILP) that determines optimization of multiple sink positions and duration of their sojourn time. Different from other studies this approach aims at maximizing lifetime rather than minimizing energy consumption. The pattern of the locations visited by mobile sinks ensures minimum number of hops to the other nodes. The study also showed that deployment of ILP for the whole network achieves longer lifetime rather than ILP for a network divided into different clusters. The paper in [40] proposed a virtual moving of multiple sinks to avoid complicated and higher energy costs due to physically moving the multiple sinks. However, excessive number of sinks is used to re-schedule activation of subset of these sinks. Active set of sinks turn on long-range communication, while passive set perform regular role of sensor nodes. The rescheduling of transmission duty cycle of long-range communication increases the degree of evenly distributing energy consumption.

\subsection{Traffic Routing}

A number of mechanisms for traffic route election that address the problem of non-fair energy consumption in critical zones are discussed in the following subsections.

\subsubsection{Heuristic route selection}

Heuristic strategy is based on a function that estimates cost of paths from source node to destination node[41-43]. Each node is assigned a heuristic value and the node with smallest function value is selected to be the next node in the path. The heuristic value changes are infrequently because most of sensor nodes are statics, and only then is the value broadcasts to other node. Little overhead is 
introduced by the transmission of heuristic value. Heuristic route-finding algorithm efficiently adjusts routes, particularly when unpredictable topological changes occur [44].

The paper in [15] proposed a heuristic routing technique to alleviate energy hole problem by considering energy consumption and distance to destination. In each corona zone an aggregator node is nominated as a header and forwards its data to corresponding header at lower corona towards the sink node. Selection of header is made on the basis of remaining energy and distance from cluster head to sink node. The authors in [45] proposed a Heuristic Load Distribution algorithm (HeLD) multipath routing to achieve equal energy consumption rate among nodes of the same depth which subsequently saves cost of transmission. A sensor node compares its parents in pair wise manner and switch to other parents if cost of route of current parent is higher than the others. Traffic flow is controlled by a central agent with full knowledge of the network topology. Optimum traffic rate of each link is based on awareness of parents' input traffic rates by averaging the number of received packets during a time interval. The paper in [46] proposed a heuristic function to find energy efficient forwarding path that protects the network from partitioning. The energy aware heuristic-based routing (EAHR) protocol selects route from source to destination node on the basis of smaller values of heuristic functions. This function combines a number of parameters; longevity factor which is residual initial energy ratio and cost to the sink which is a combination of residual energy and distance to the sink node.

\subsubsection{Greedy route selection/transform}

There are various policies for greedy forwarding to select a candidate neighbor node that is in the line-of-sight towards the destination; they are such as position, hop count and node energy [47-48]. The authors in [49] proposed Adaptive Greedy-Compass Energy-Aware Multipath (AGEM) which gives a score to each one-hop neighbor and select a set with smallest angular offset from a virtual line toward the destination. This set of neighbors ensures multiple paths from a source node. The routing decisions are performed based on a limited and localized knowledge of the network. A packet with the smallest number of hops is forwarded through the neighbor nodes with higher energy consumption and vice versa where a packet with the highest number of hops is forwarded through neighbor nodes with lowest energy consumption. Multipath capabilities ensure uniform energy consumption and meets the delay and packet loss constraint. The algorithm confirms the occurrence of an energy a hole when it fails to find a set of neighbors, it accordingly forwards the responsibility to the neighbors nearer to the source node. The paper in [50] proposed a supervisory routing control for dynamic load balancing. This greedy algorithm avoids early death of relatively overloaded nodes that cause network partition. Traffic load of nodes is monitored and overloaded nodes are identified. Monitoring is done by means of innetwork path tagging which is deterministic and light-weight. Child of the overloaded node is then instructed to switch from its parent node. The instruction is sent via the Sink to the child node while acknowledgement for successful switching is sent back from child to sink. The research in [51] proposed Hole Avoiding In advance Routing protocol (HAIR) where local information about one-hop neighbors is maintained. If a hole is detected by a node, then neighbors are informed by "a hole detection" message via "push" action. Accordingly, neighbors reroute their data traffic to another parent node according to traditional geographic routing protocols where distance is considered for the selection of the next hop is the shortest distance to the destination.

\subsection{Hybrid mechanisms}

Hybrid is the integration of a number of different tactics of previously discussed remedies. In the paper [52] proposed this approach which integrates power control and exponential node distribution to alleviate hole problems. It is based on node residual energy where each node closest to the sink decides whether to send through intermediate nodes or directly to the destination. A sub-balanced energy consumption via non-uniform node distribution is integrated with greedy routing to increase the efficiency of energy balance. Greedy routing selects a parent node or send its data directly to the base station. The authors in [53] proposed dynamic routing adapted to changes in external environment 
to mitigate hole problem. To reduce energy consumption of dynamic routing, routing table reconfiguration is restricted to only the changed parts of the topology.

Dynamic traffic routing aims at distributing evenly energy consumptions over the entire network. Integrating with moving mobile sink heuristically according to a threshold of average residual energy surrounding the sink (ARES), improves energy balance and hence extends network lifetime. The research in [54] proposed Unattended Mobile Sensor Networks through Autonomous Sensor Redeployment laid on non-uniform node distribution in different coronas. The vicinity area of interest is divided into equal width coronas. Mobile nodes are allowed to move between adjacent coronas to readjust node distribution required in intended corona avoiding high energy consumption. The study in [55] proposed energy hole countermeasure that merges two strategies of node deployment; multiple sinks and sensor nodes distribution, while the density of sensor nodes is increased in critical zones. The study examined the number of sinks to estimate the number of sensors in hole zones. Integration of node distribution with multiple sinks balances energy consumption, and as a result, extends network lifetime.

\section{Energy Hole Adaptability to Tree Topology Feature}

This section analyses energy consumption in energy hole zones closest to the sink nodes and the impact of different tree topology constructions. Energy consumption based on radio energy model in Figure 3 is discussed in [56].

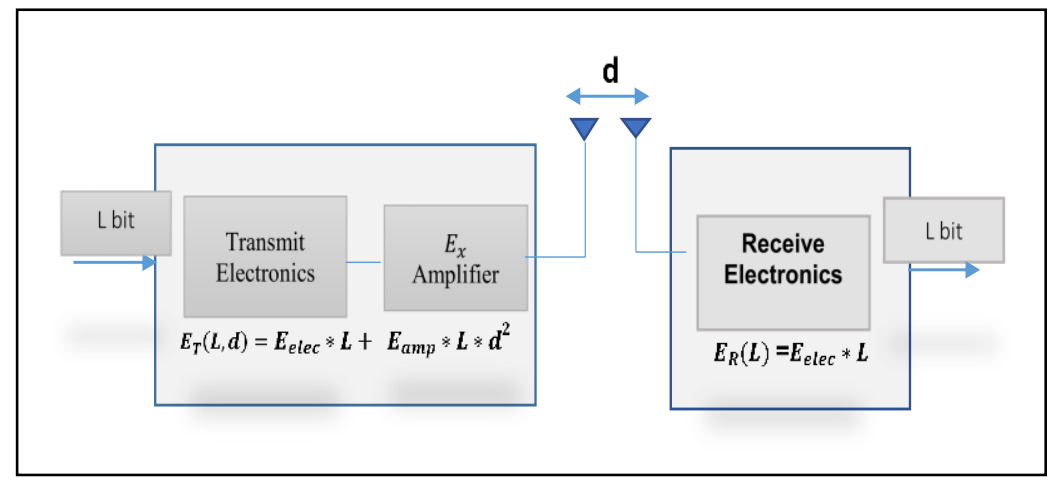

Figure 3. Basic sensor node energy dissipation model

In this paper, relaying sensor node (RN) is characterized with one child node or one sensor chain. As shown in Figure 4, nodes at the lower levels of a tree (closer to the sink node) which join a number of upper-level branches, tend to be more energy exhausted compared with nodes at the upper levels.

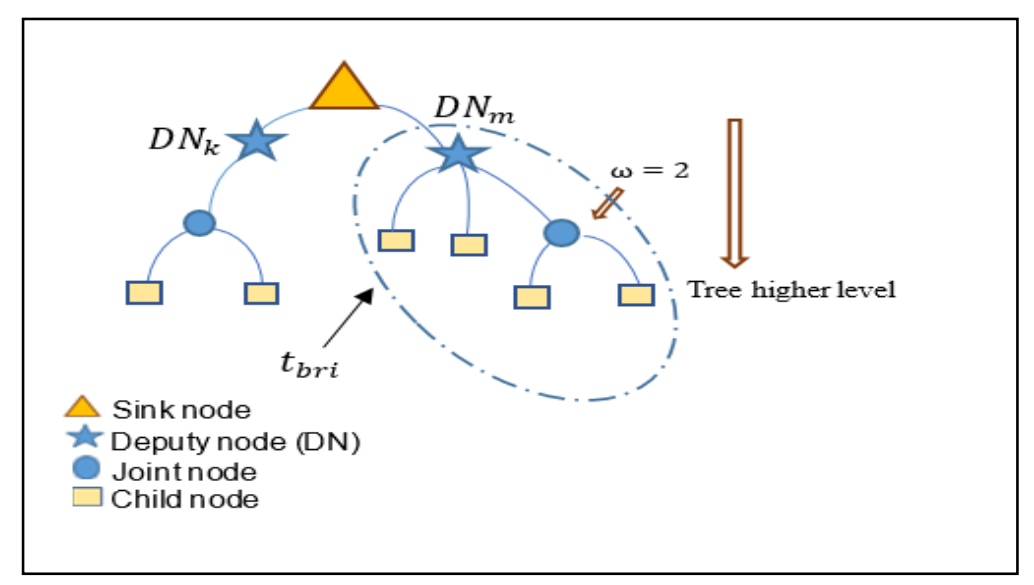


Figure 4. Tree topology of joint nodes

An analysis is made on such joint nodes, denoted by zeta $\zeta$ and how the distribution of $\zeta$ impacts the diversity of variations in energy dissipation amongst all subtrees in a tree topology. A joint node is characterized by more than one child node or more than one chain of descendant nodes. The energy consumption behavior is strictly correlated to the node association manners in tree topology alongside the traffic route that satisfies the performance requirement. Energy consumption in RN associated to a subtree can be given as a function of transmission and reception of packets [57], given as follows:

$E_{T t_{b r i}}(L, d)=\sum_{l=1}^{R N}\left[E_{l}^{a}+E_{l}^{b}\right]$

where

$R N$ is the entire number of sensor nodes in tree topology.

$$
\begin{gathered}
E^{a}=E_{\text {consuTx }}(\mathrm{L}, \mathrm{d})=E_{\text {elec }} * L+E_{\text {amp }} * L * d^{2} \\
E^{b}=E_{\text {consuRx }}(\mathrm{L})=\boldsymbol{E}_{\text {elec }} * \mathrm{~L}
\end{gathered}
$$

$E_{\text {consu }}$ is the energy consumed by a sensor node to transmit and receive data.

$R_{x}$ and $T_{x}$ denote the function of reception and transmission of packets, respectively.

$\mathrm{L} \quad$ is the number of bits in a packet.

$\mathrm{d}$ is the distance between two communicating sensor nodes,

$E_{a m p}$ is the sensor node amplification energy for transmitting.

$E_{\text {elec }}$ is the sensor node circuit energy consumption.

$\zeta$ is not the same as with relaying node (RN) in its rate of energy consumption pattern but is similar in that the amount of energy consumption is inversely proportional to its distance from the sink node. From Equation (3), the increase in energy consumption $E^{b *}$ to receive packets in a joint node $\zeta$ is based on the number of downstream traffic $\omega$,

$$
E^{b *}=\sum_{\mathrm{j}=2}^{\omega} E_{j}^{b}
$$

Where $\omega$ is the number of child nodes or chains of leaf nodes interconnected to joint node $\zeta$.

Hence, while the total number of nodes is fixed in a tree topology, the diversity and variations in energy consumption $\left(E_{\text {ConsuRx }}\right)$ due to packet receive is influenced by the number of joint nodes in each subtree out of its total number of nodes. The total energy consumed by the number of nodes, $n$ during packet reception in a subtree $t_{b r i}$ can be expressed as follows:

$$
E_{\text {tot }_{R x t_{b r i}}}(L)=\sum_{i=1}^{n} E_{i}^{b}
$$

If $\mathrm{h}$ is the number of relay nodes in a subtree not including joint nodes, then the total energy consumed by a sensor node to receive a packet can be given as:

$$
E_{\text {tot }_{R x t} t_{b r i}}(L)==\sum_{\mathrm{k}=1}^{h} E_{k}^{b}+\sum_{\mathrm{m}=1}^{\zeta} E_{m}^{b *}
$$

Accordingly,

$$
E_{\text {tot }_{R x t} \text { bri }}(L)==\sum_{\mathrm{k}=1}^{h} E_{k}^{b}+\sum_{\mathrm{m}=1}^{\zeta} \sum_{\mathrm{j}=2}^{\omega} E^{b j}
$$

Where the second part of Equation (7) is the total energy consumed, influenced by the number of interconnected child nodes or chains of leaf nodes $\omega$ ) in joint nodes $(\zeta)$. The smallest number of interconnected children or chains of leaf nodes is $\omega=2$.

It is important that we compare variations in energy consumptions between the primary route and that of alternative routes, particularly when more than one subtree $\left(t_{b r i}\right)$ are interconnected to a

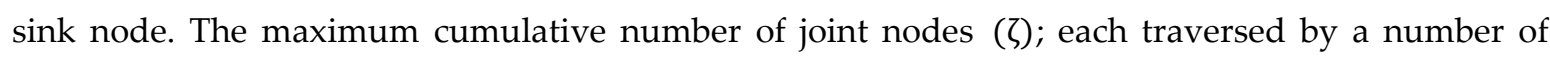


packets proportional to the values of $\omega ; \sum_{\mathrm{m}=1}^{\zeta} \sum_{\mathrm{j}=2}^{\omega} E^{b}{ }_{m j}$, results in variations in energy consumption among the entire subtrees that interconnect to a sink node and consequently influence the energy consumption balance across the network. This is because the rate of energy consumption by such a node is different from that of relay node $(\mathrm{h})$, whether it is a child or a leaf node in the same subtree. Accordingly, the second part of Equation (7) can be used to examine the level of energy consumption balance among multiple subtrees. Standard deviation (STD) of energy consumption is an important energy balance indicator used to explore fairness and even performance among a number of $t_{b r i}$. In such a network, the minimum STD indicates that energy consumption of all the nodes is close to each other. The average energy consumption in a subtree; $\overline{E_{t o t_{R x, T x}\left(t_{b r l}\right)}}$, where the sum of joint nodes $\zeta$ and the remaining regular nodes $h$ equals to the total number of node $n$ :

$$
\bar{E}_{\text {tot }_{R x, T \times\left(t_{b r l}\right)}}(r)\left\{\begin{array}{c}
\text { multihop disjoint node }\left[\frac{1}{n} \sum_{i=1}^{n}\left[E_{i}^{a}+E^{b}{ }_{i}\right]\right], \\
\text { multihopSubtree }\left[\frac{1}{h} \sum_{\mathrm{k}=1}^{h}\left[E_{k}^{a}+E_{k}^{b}\right]\right]+ \\
{\left[\frac{1}{\zeta}\left[\sum_{m=1}^{\zeta} E_{m}^{a}+\sum_{\mathrm{m}=1}^{\omega} \sum_{\mathrm{j}=2} E^{b}{ }_{m j}\right]\right]} \\
\text { if number of } \zeta \geq 1
\end{array}\right.
$$

In this section, the correlation between energy performance in tree topology to the sensor nodes' association manners is analyzed using MATLAB, particularly the influence of energy hole zones by joint nodes. The performance is explored under different tree configurations in terms of the distribution of joint nodes $\zeta$, number of source nodes in subtree $n$, and at different packet generation rates $(\mathrm{R})$. Table 1 lists the important parameters and their corresponding values that have been considered in this assessment.

Table 1. Parameter setting for tree topology analysis using MATLAB

\begin{tabular}{l|c|c}
\hline Parameter & Value & Units \\
\hline Number of nodes & 90 & nodes \\
\hline Number of sinks & 1 & nodes \\
\hline Packet size & 56 & Byte \\
\hline Packet rates & 1 & Packet/sec \\
R1 & 2 & \\
R2 & 3 & \\
R3 & 3 & nodes \\
\hline Number of deputy nodes, DNs & $30,37,23$ & nodes \\
\hline Subtree1, subtree2, subtree3 & & \\
\hline Subtree1,2, and 3 of, & $4,6,4$ & $\zeta$ \\
First tree topology & $6,6,6$ & \\
Second tree topology & & \\
\hline
\end{tabular}




\begin{tabular}{l|l|l}
\hline & & \\
& & \\
\hline Case1 & R1, R1, R1 & \\
Case2 & R2, R2, R3 & Packet rate \\
Case3 & R1, R3, R3 & \\
\hline
\end{tabular}

Figure 5 depicts a wireless sensor network distributed over an area of $500 \mathrm{~m}$ by $500 \mathrm{~m}$ as illustrated by the axes. Two different topologies, identied as Topology A and B, can be discerned from this arrangement as will be described next. The tree topology is constructed with equal number of node associations, which is 90 nodes, and each of the 2 topologies consists of 3 subtrees which are interconnected. They are labeled as Subtree 1,2 and 3 respectively as shown in the figure.

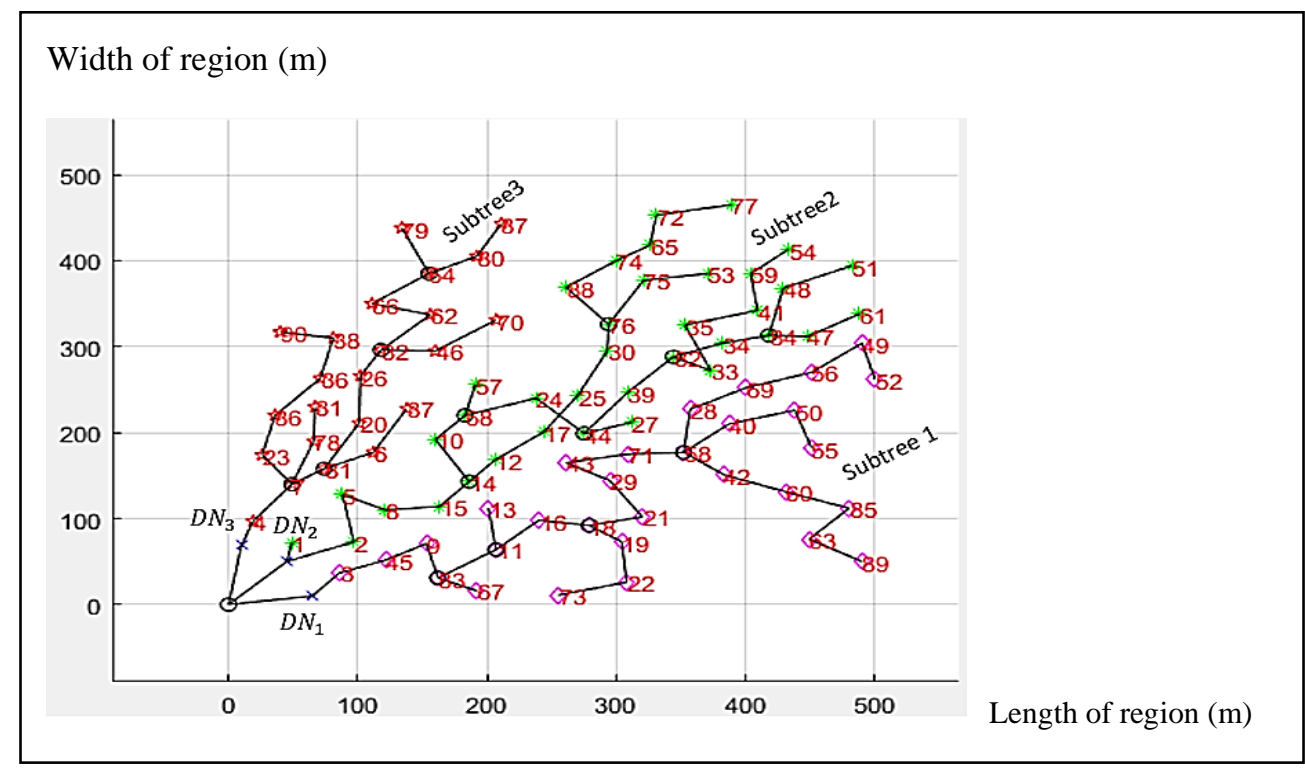

Figure 5. The topology of the sink-rooted tree with 3 subtrees

In Topology A, subtrees 1 and 3 each has a maximum of four joint nodes (4ל), while Subtree 2 has six joint nodes (6ל). Subtrees 1, 3 and 2 have 30, 23 and 37 sensor nodes, respectively. For Topology $B$, there are equal distribution of joint nodes in each subtree; that is, six for each subtree (6ろ). Both tree topologies (Topology A and B) are next examined under several cases with traffic generation rates R; Case 1, 2 and 3.

In Case 1, all the source nodes of all subtrees are equal in their traffic generation rates, while in Case 2, the data rate of the source nodes of Subtrees 2 and 3 are made to be double and triple to that of Subtree 1, respectively.

Figure 6 depicts the energy deviation at the sink's energy hole in these trees. For each of the Cases, Topology B experiences a smaller energy consumption deviation compared to Topology A. The results indicate that the energy distribution of nodes around a sink energy hole is influenced by the distribution of joint nodes $\zeta$ and the traffic generation rate of source nodes. It could be seen that the number of joint nodes $\zeta$ in subtree 2 is 6 ; this is greater than that in subtree 1 , which has 4 joint nodes. These elements are vital in managing energy consumption pattern. 


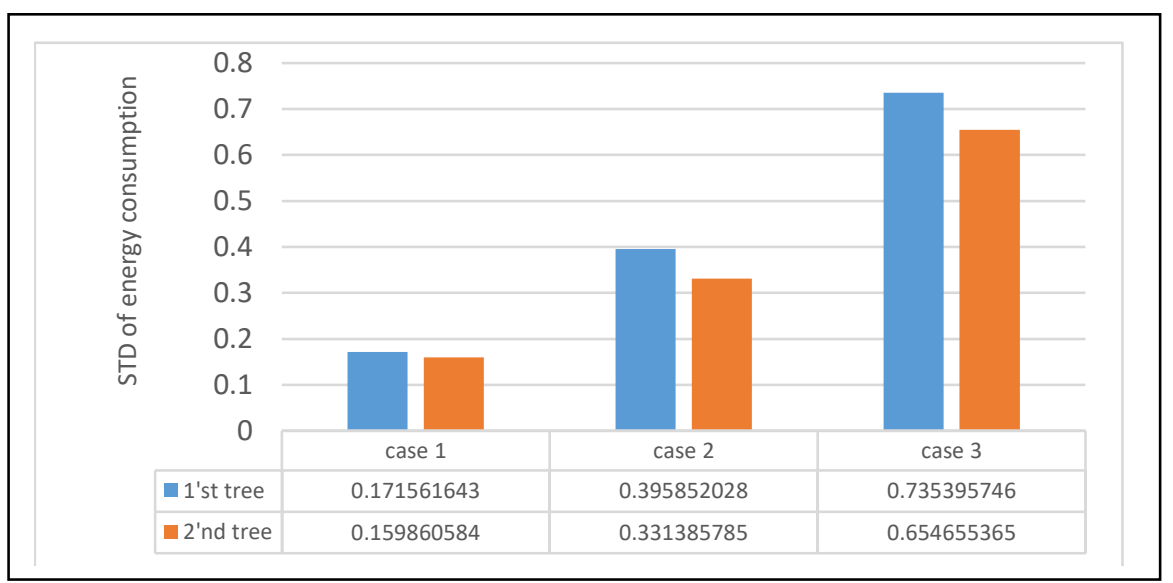

Figure 6. Energy balance at sink hole

\section{Hole Status Awareness and Routing Decision}

Further attention is needed to protect the deputy nodes against energy hole formation. Robust energy awareness-based routing decision plays an important role in obtaining fair energy consumption amongst deputy nodes throughout the communication period. The proposed routing metric $\boldsymbol{Q}$, upon which the decision of traffic routing rested upon, correlates energy status in critical zones, denoted by $\widehat{\boldsymbol{Q}}_{\boldsymbol{k}, \boldsymbol{m}}$, with vital topology construction features, i.e the joint nodes, $\boldsymbol{\zeta}$. The routing decision needed to be made for the next transmission round " $r$ " is; whether to keep data traffic forwarding through the primary tree or switch to an alternative route.

Figure 7 illustrates a tree with two interconnected subtrees rooted on the deputy node (DN) labeled as $\mathrm{k}$ and $\mathrm{m}$ respectively with their respective energy pattern $\widehat{\boldsymbol{Q}}_{\boldsymbol{k}, \boldsymbol{m}}$. The dotted line in the figure indicates that the source node can switch its traffic route to the parent node that is interconnected to a different path. Energy routing metric $\boldsymbol{Q}$ of parent node candidates are first compared to each other by the source node for taking the responsibility of traffic delivery to the sink node.

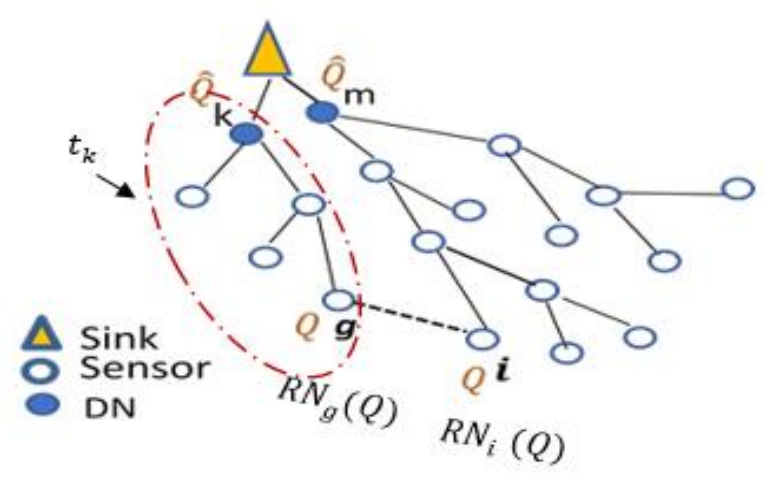

Figure 7. Sink-rooted multi subtree

The core assumptions of this proactive energy routing metric in tree topology with a single sink are as follows:

- Any change in either tree depth or the rule of sensor nodes association will determine the estimated number of joint nodes,

- A joint node encounters more data traffic as it gets closer to the sink (in hop counts). 
The source node is aware of its local information in terms of short hop count and cumulative number of $\boldsymbol{\zeta} . \quad \boldsymbol{S} \boldsymbol{H} \boldsymbol{C}_{\boldsymbol{T}_{i}}^{\boldsymbol{k}, \boldsymbol{m}}$ is the short hop count of the source node to the sink node through a corresponding deputy node, $\boldsymbol{D} \boldsymbol{N}_{\boldsymbol{k}, \boldsymbol{m}}$ and $\boldsymbol{\zeta}_{\boldsymbol{T}}^{\boldsymbol{k}, \boldsymbol{m}}$ refers to the number of joint nodes that are traversed by data packets in a tree $\boldsymbol{T}_{\boldsymbol{i}}$ to the deputy nodes $\mathrm{k}$ or $\mathrm{m}$ in the sink node. The number of joint nodes $\boldsymbol{\zeta}$ is accumulated from the lowest level of the tree $\boldsymbol{t}_{\boldsymbol{b r}(\boldsymbol{k}, \boldsymbol{m})}$ (nearer to the sink) to its highest level where the source node is located. $\boldsymbol{\zeta}_{\boldsymbol{T}_{i}}^{\boldsymbol{k}, \boldsymbol{m}}$ aids the routing algorithm to estimate the node chains interconnected to a possible path within a sub tree. Also, $\boldsymbol{S H}_{\boldsymbol{T}_{\boldsymbol{i}}}^{\boldsymbol{k} \boldsymbol{m}}$ is a short hop count to deliver traffic to the sink node and it is one of the parameters which influence energy consumption in tree topology, particularly, the number of packets received in each deputy node, DN. Accordingly, these parameters are route feature in tree topology denoted by the symbol $\boldsymbol{N}_{\boldsymbol{T}_{\boldsymbol{i}}}$ considered in routing decision as shown in;

$$
N_{T_{i}}=\zeta_{T_{i}}^{k, m} * S H C_{T_{i}}^{k, m}
$$

In our proposed EBEHA-T, the routing metric for making routing decision for the next round is expressed as;

$$
Q=\frac{\widehat{Q}(k, m)}{N_{T_{i}}}
$$

This is the ratio between the energy level in the considered deputy nodes ( $\mathrm{k}$ or $\mathrm{m}$ ) to the product of number of joint nodes and hop count.

The decision to determine whether the selected deputy node is going to be $\mathrm{k}$ or $\mathrm{m}$ as shown in Figure 6 is expressed as:

$$
\widehat{\boldsymbol{Q}}(\boldsymbol{k}, \boldsymbol{m})=\frac{d}{r}\left(\boldsymbol{E}_{\text {sink }}-\boldsymbol{E}_{\text {res }(\boldsymbol{k}, \boldsymbol{m})}\right)
$$

Where $\boldsymbol{E}_{\text {res }(\boldsymbol{k}, \boldsymbol{m})}$ is the residual energy of an intended DN, referenced to sink node to enable this sink to evaluate DNs every transmission round $\mathrm{r}$ and denoted by $\frac{d}{r}$. The energy status at critical zones, $\widehat{\boldsymbol{Q}}(\boldsymbol{k}, \boldsymbol{m})$ corresponding to $\boldsymbol{D} \boldsymbol{N}_{\boldsymbol{k}, \boldsymbol{m}}$, is announced through the sink's beacon.

EBEHA-T is designed to be an energy status $\boldsymbol{Q}$ metric-based routing decision as illustrated in Figure 8. At each transmission round, the source node recognizes all the potential parent nodes by way of some "hello" messages. These parent nodes are interconnected to different deputy nodes in the hole zone. The respective source nodes then perform a comparison of their respective energy statuses to identify an energy-efficient route. Parents with the best energy status $\boldsymbol{Q}$ will be selected to be the traffic forwarding node since it belongs to the most appropriate subtree in terms of root node's energy consumption variation with respect to route communication costs towards the sink node.

It is important to use routing metric $\boldsymbol{Q}$ to determine the quality of the different routes. Thus, referring to the flowchart in figure 8, when the routing metric $\boldsymbol{Q}\left(\boldsymbol{t}_{\boldsymbol{m}}\right)$ of a parent node in subtree $\boldsymbol{t}_{\boldsymbol{m}}$ is lower than a routing metric $\boldsymbol{Q}_{\boldsymbol{g}}\left(\boldsymbol{t}_{\boldsymbol{k}}\right)$ of the primary parent node $\boldsymbol{R} \boldsymbol{N}_{\boldsymbol{g}}$ in the subtree $\boldsymbol{t}_{\boldsymbol{k}}$, then the source node $\boldsymbol{R} \boldsymbol{N}_{\boldsymbol{i}}$ switches its traffic route to $\boldsymbol{R} \boldsymbol{N}_{\boldsymbol{g}}$ instead of primary parent node. This would be the case if the decision is left to auto-routing operation. In this case the relay node will end up in a deputy node having a higher residual energy compared to that of its primary route. Also, if the primary parent is higher than or equal to, the source node uses the primary traffic route since its deputy node has a higher residual energy or does not have a serious variation in energy consumption pattern among DNs in critical zones. 


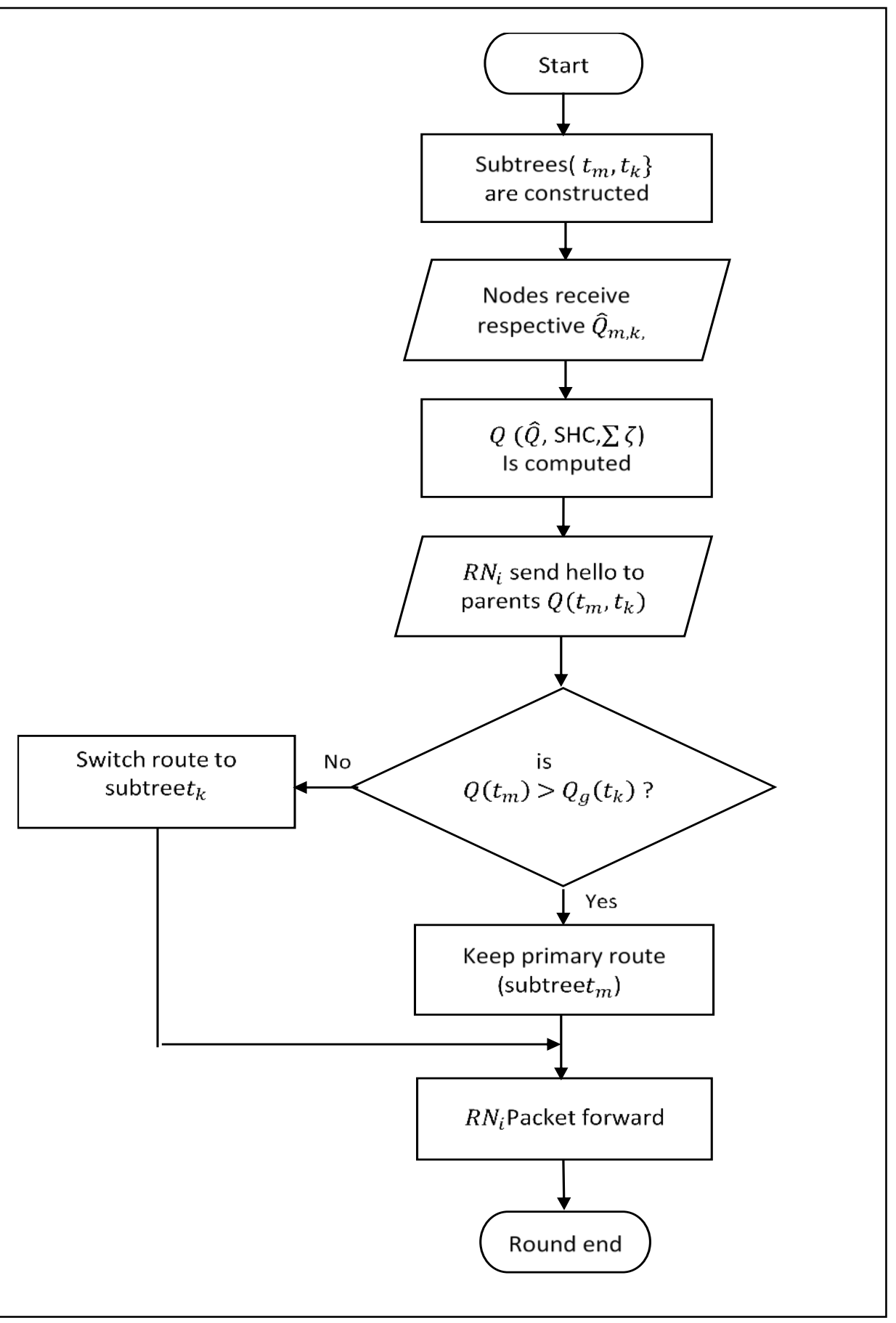

Figure 8. Flowchart of proactive energy routing metric-based traffic routing

The decision is left to auto-routing operation. In this case the relay node $\boldsymbol{R} \boldsymbol{N}_{\boldsymbol{g}}$ will end up in a deputy node having a higher residual energy compared to that of its primary route. Also, if the energy metric $\boldsymbol{Q}\left(\boldsymbol{t}_{\boldsymbol{m}}\right)$ of the primary parent is higher than or equal to $\boldsymbol{Q}_{g}\left(\boldsymbol{t}_{\boldsymbol{k}}\right)$ in subtree $\boldsymbol{t}_{\boldsymbol{k}}$, the source node uses the primary traffic route since its deputy node have a higher residual energy or does not have a serious variations in energy consumption pattern among DNs in critical zones. Accordingly, it is crucial that at each transmission period, the sink nodes update the energy status amongst its deputy nodes and that the source nodes are aware of the tree topologies construction features such as hop count and joint nodes. 


\section{Simulation Results and Discussions}

This section presents the performance of our proposed EBEHA-T routing algorithm compared against Randomized Switching for Maximizing Lifetime (RaSMaLai)[58]. RaSMaLai has been selected as the benchmark method as it is one of the latest works in the literature that addresses the energy hole problem in a tree topology-based traffic routing. It aims to maximize the lifetime of a sensor network by using a routing decision considering load balance parameters to switch from original paths to other paths with lower load.

Several simulations of these schemes have been carried out using OmNet++ which is a popular open source network simulation package. Standard radio parameters and energy dissipation model has been implemented. Table 2 presents the simulation parameters used to assess the performance of the tree topology.

Table 2 Simulation parameters used in OmNet++ v4.62.

\begin{tabular}{lcr}
\hline Parameter & Value & Units \\
\hline Number of static nodes & $30-75$ & nodes \\
\hline Number of static sinks & 1 & nodes \\
\hline CS1 and CS2 & 3 and 4 & DN \\
\hline Initial Energy & 0.5 & joule \\
\hline Circuit energy to transmit /receive (Tx/Rx) & 50 & nJoule/bit \\
\hline Energy of amplification & 10 & Pjoule/bit/m2 \\
\hline Packet rate & & \\
R1 & 1 & Packet/sec \\
R2 & 0.5 & \\
\hline MAC & CSMA/CA & \\
\hline
\end{tabular}

Different network situations which we refer to as case studies (CS) have been modelled to study the performance of EBEHA-T and RaSMaLai under different tree topologies. The cases are basically referred to a single-sink tree topology varying in sink energy hole size from four deputy nodes (4DN) to eight deputy nodes (8DN), and total number of sensor nodes from 30 to 75 for CS1 and CS2, respectively.

\subsection{Average energy consumption}

Figure 9 and 10 show the average consumed energy for the two cases -- CS1 and CS2, and under different traffic generation rates R1 and R2, respectively. The multiple subtree structure that our proposed EBEHA-T is laid on enables source nodes to explore available short routes which subsequently contributes to a reduction in communication costs. From the simulation run shown in figure 9, it can be seen that EBEHA-T shows a lower energy consumption behaviour compared to that of RaSMaLai, by approximately $50 \%$. This observation indicates that the awareness of energy pattern $\widehat{\boldsymbol{Q}}$ as well as joint nodes $\zeta$ together play a dominant role in managing the energy consumption of the network. In Figure 10, for case study CS2, it is shown that the energy consumption by EBEHA-T is $54 \%$ to that of RaSMaLai. Thus, regardless of underlying topology, EBEHA-T has shown to be an effective mechanism for energy savings in tree based wireless sensor networks. 


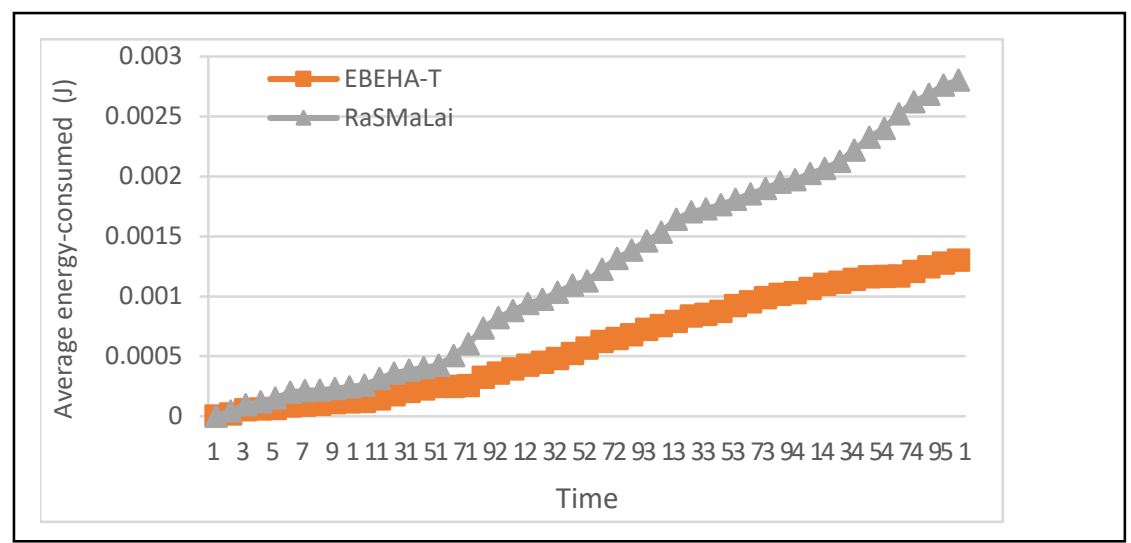

Figure 9. Network-average consumed energy of EBEHA-T against RaSMaLai with small energy hole, CS1

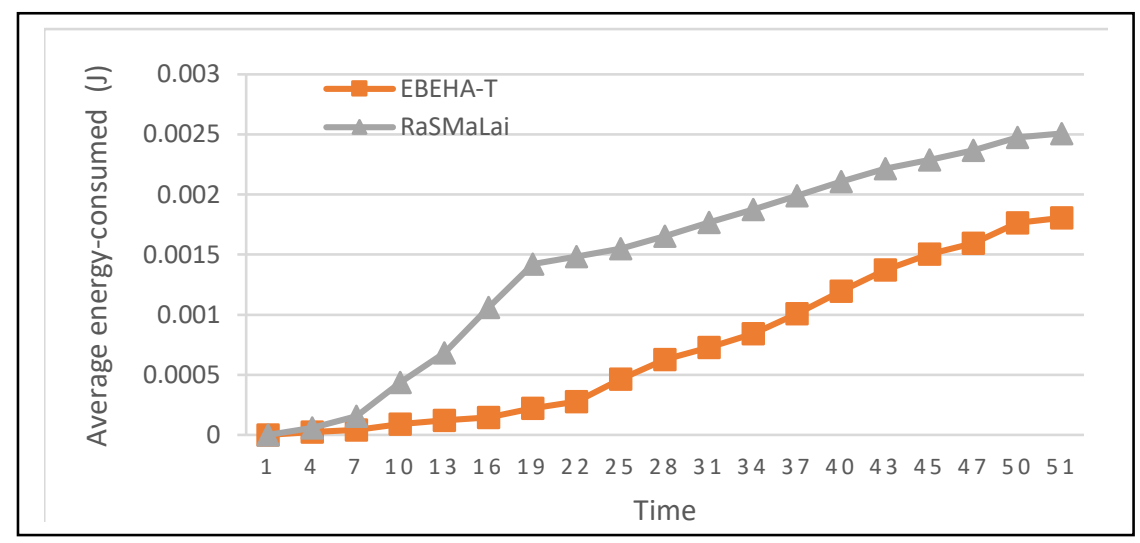

Figure10. The average consumed energy of EBEHA-T and RaSMaLai in a large tree topology and energy hole, in CS2

\subsection{Network lifetime}

The energy performance in EBEHA-T has been further examined through fair energy exhaustion across the entire network. The small standard deviation (STD) of the energy consumed by the nodes across the entire network indicates a decrease in the deviation of energy consumption among sensor nodes. This implies that there is an enhancement in fair energy usage amongst sensor nodes in the network. Figure 11 and 12 show that EBEHA-T achieves enhancement in energy balance of approximately $78 \%$ and $62 \%$, respectively compared against RaSMaLai under case study CS1; and $81 \%$ and $85 \%$, respectively under case study CS2, with packet generation rates R1 and R2. Updated image of energy consumption pattern amongst deputy nodes $\mathrm{DN}$ is considered as an important parameter for energy awareness by distant source nodes in order to make their routing decisions. 


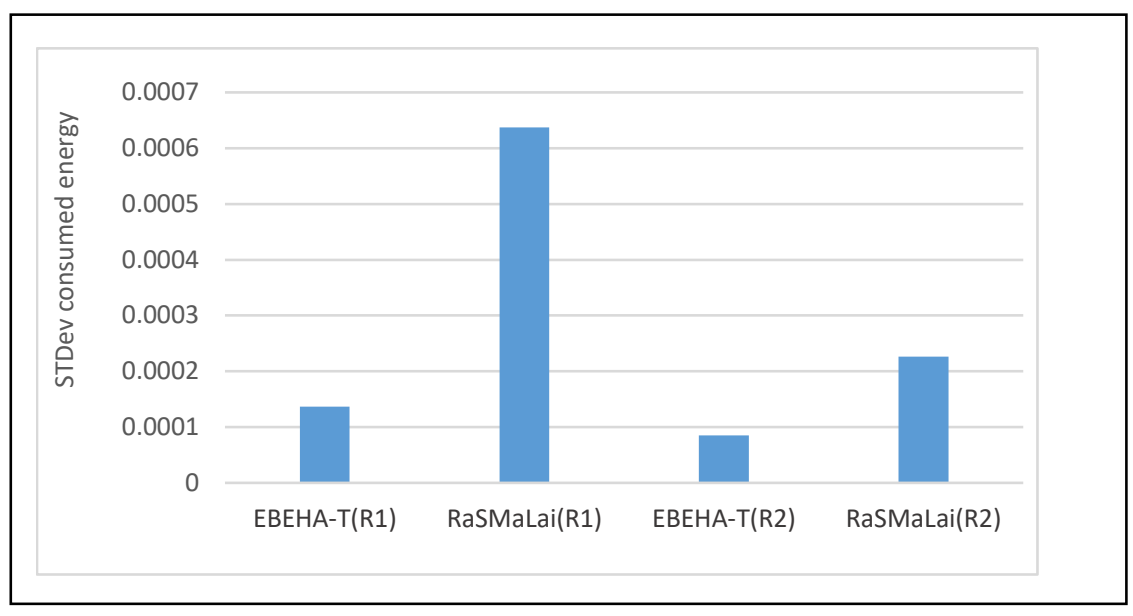

Figure 11. Sink hole's energy balance of EBEHA-T against that of RaSMaLai with small energy hole and under different rate of packet generation, CS1

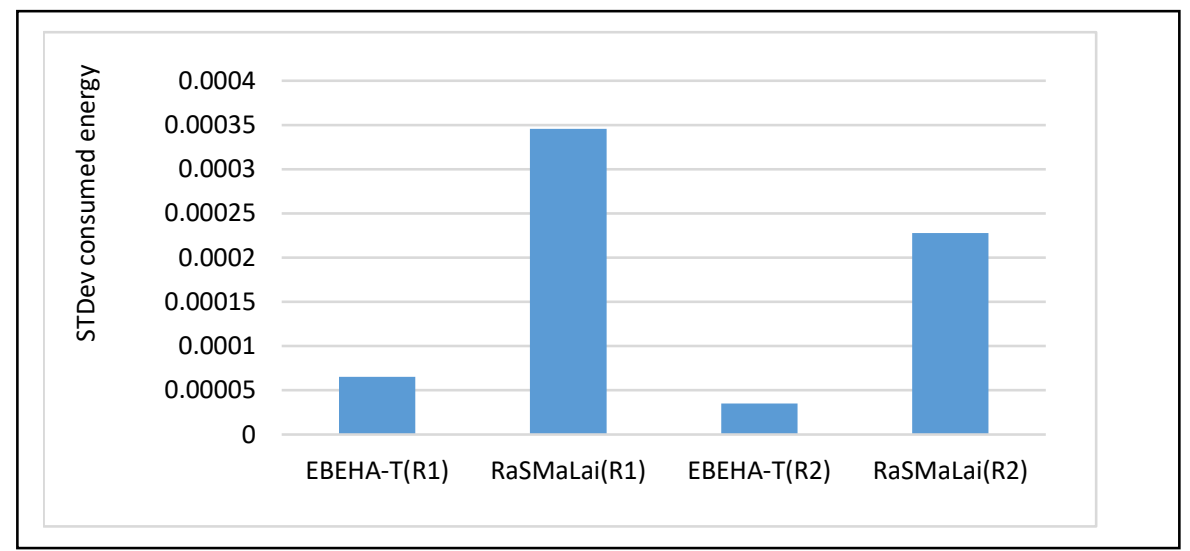

Figure 12. Standard Deviation (STD) of consumed energy for EBEHA-T compared against RaSMaLai with large energy hole and under different rates of packet generation, CS2

The outcome of energy balance and savings across the entire sensor network and reduction in deviation in energy consumption at hole zones, is that the network lifetime is extended significantly. This is a desireable property of sensor networks as the network is expected to be left operating over a long period of time without human intervention. Network lifetime is defined as the length of time the network lasts until the first node dies. Figure 13 and Figure 14 show the operation of EBEHA-T and RaSMaLai for a tree topology over the length of simulation time. From these figures, it is clear that RaSMaLai experiences a relatively faster node death at 30 and 34 simulation times, respectively whereas EBEHA-T shows an extended lifetime beyond the furthest range of simulation time performed in this simulation exercise. 


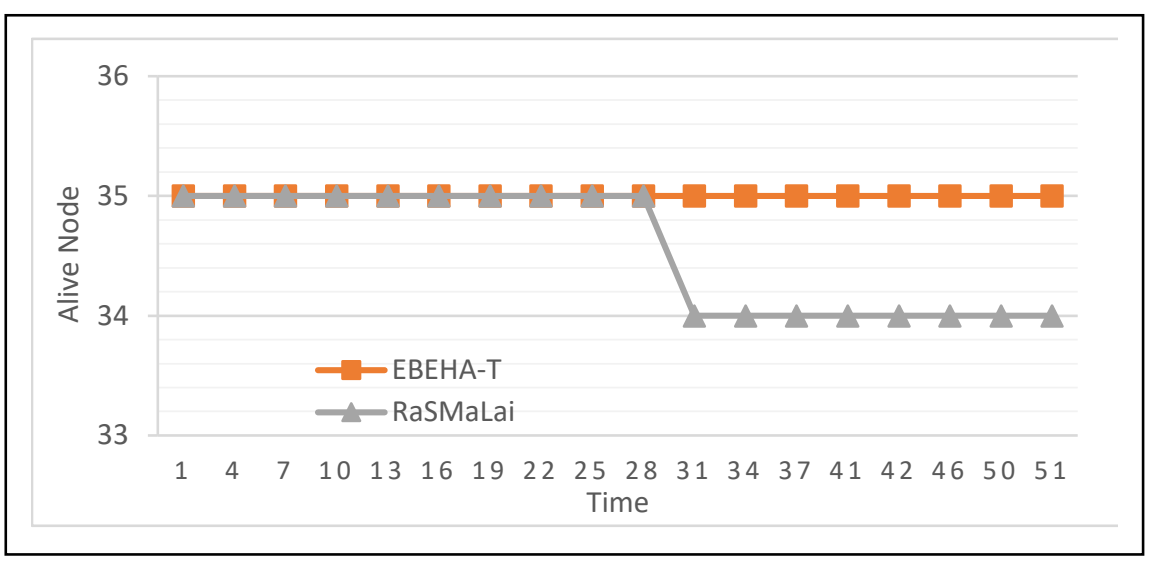

Figure 13. Sensor Network lifetime with EBEHA-T and RaSMalai under traffic generation rate R1 of 1 packet/s, and network case CS1

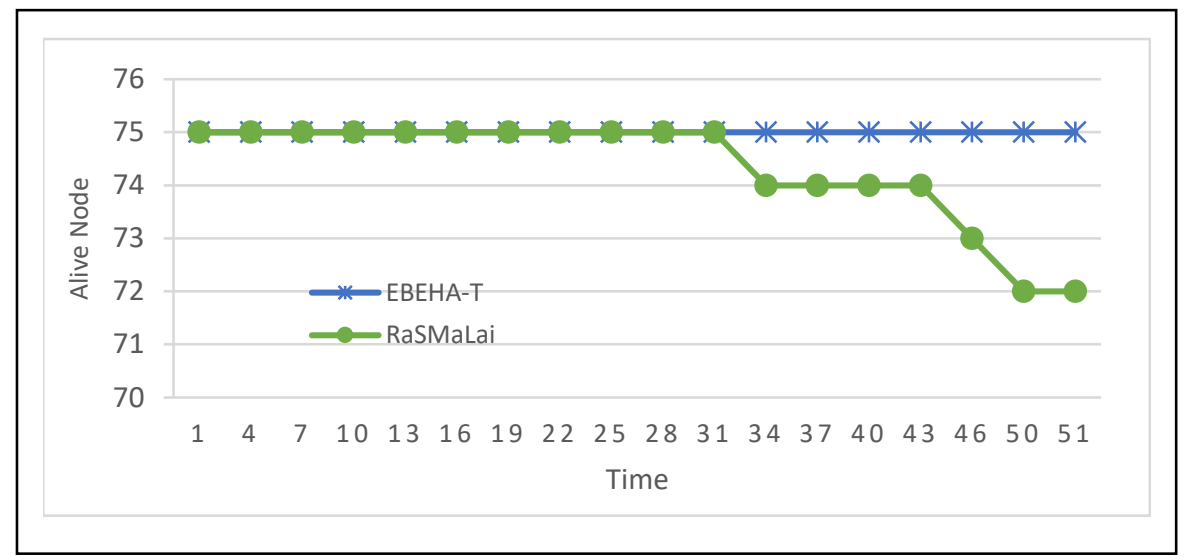

Figure 14. Sensor Network lifetime with EBEHA-T and RaSMaLai under traffic generation rate R2 1 packet/2s, and network case CS2

\subsection{Packet delivery ratio}

Another important performance assessment of a tree based wireless sensor networks is its packet delivery ratio (PDR). This is defined as the ratio between the number of packets that are successfully delivered to the sink node, against the total packet generated by the source nodes. Figure 15 shows PDR for EBEHA-T and RaSMaLai for different traffic-generating rates under case study CS1. Again, EBEHA-T shows superior performance in terms of PDR of approximately 0.82 against 0.57 for RaSMaLai; while with packet rate R2, the PDRs are 0.97 and 0.64 for EBEHA-T and RaSMaLai, respectively. Thus, clearly EBEHA-T shows a higher level of network reliability demonstrated by the higher packet delivery ratio, PDR, for both packet generation rates. 


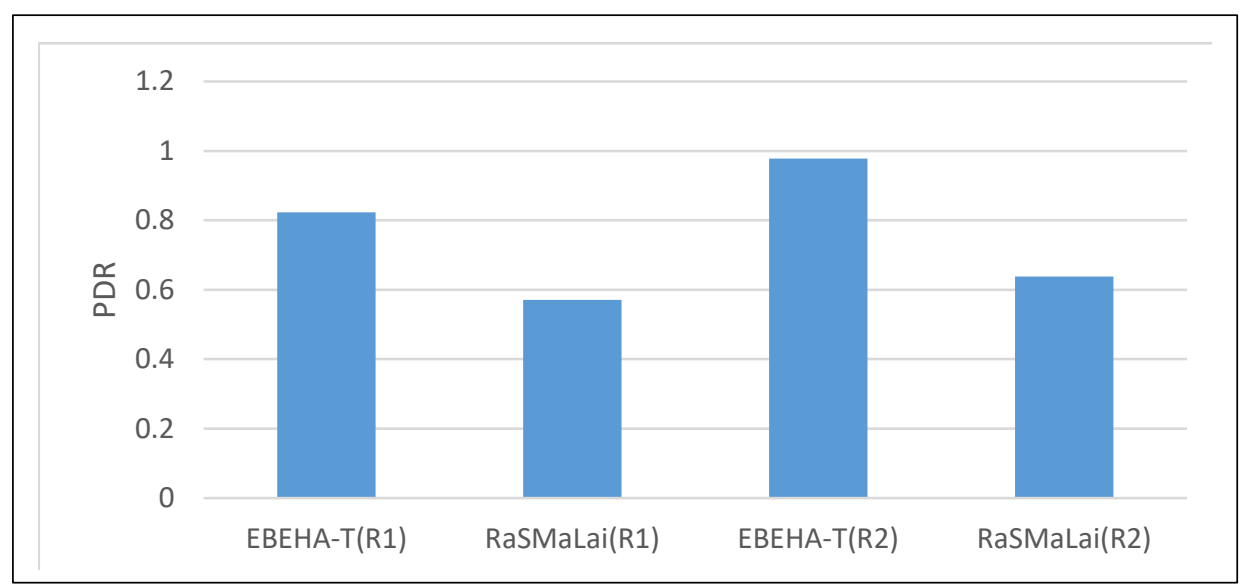

Figure 15. Packet delivery ratio (PDR) of EBEHA-T and RaSMaLai under different rates of packet generation, and network case CS1

\section{Conclusion and Future Works}

This paper addressed the problem of energy hole formation at a wireless sensor network sink. The energy hole causes isolation of the sink node which subsequently prevents data from being delivered to the remote end and network partitioning which isolates the network into several small disjoint networks. Previous studies did not consider energy status of the sink's energy hole zones, which otherwise could aid in making routing decisions which would preclude energy hole formation, rather than retrospectively responding to a hole detection. In this paper, we considered updated energy consumption behavior amongst deputy nodes around hole zones together with estimated cumulative joint nodes, as a new energy-aware metric for our proposed EBEHA-T routing algorithm. The superior performance of EBEHA-T compared against state-of-the-art RaSMaLai verifies that its energy-aware routing decisions results in significant improvement in the sensor network's energy consumption balance, particularly amongst deputy nodes in energy hole zones. It also affords a higher reliability in terms of packet delivery to the sink node which eventually results in a prolonged network lifetime. Future studies should include protection against relatively overwhelmed traffic at some sinks in a tree topology with multiple sinks to achieve a longer network lifetime.

\section{References}

1. Chen, X.; He, C.; Jiang, L. The Tradeoff between Transmission Cost and Network Lifetime of Data Gathering Tree in Wireless Sensor Networks. IEEE Int. Conf. Commun. 2013, 1790-1794. https://doi.org/10.1109/ICC.2013.6654779.

2. Jannu, S.; Jana, P. K. Energy Efficient Algorithms to Maximize Lifetime of Wireless Sensor Networks. Conf. Adv. Comput. Commun. Informatics 2016, 63-68.

3. Jan, N.; Hameed, A. R.; Ali, B.; Ullah, R.; Ullah, K.; Javaid, N. A Wireless Sensor Networks Balanced Energy Consumption Protocol. Proc. - 31st IEEE Int. Conf. Adv. Inf. Netw. Appl. Work. WAINA 2017 2017, 730-736. https://doi.org/10.1109/WAINA.2017.122.

4. Wan, S.; Zhang, Y.; Chen, and J. On the Construction of Data Aggregation Tree With Maximizing Lifetime in Large-Scale Wireless Sensor Networks. IEEE Sens. J. 2016, 16 (20), 7433-7440. https://doi.org/10.1109/TC.2015.2512862.

5. Khan, Z. A.; Member, S.; Alrajeh, N.; Khan, M. I. On Energy Hole and Coverage Hole Avoidance in Underwater Wireless Sensor Networks. IEEE Sens. J. 2016, 16 (11), 4431-4442.

6. Abd, M. A.; Al-Rubeaai, S. F. M.; Singh, B. K.; Tepe, K. E.; Benlamri, R. Extending Wireless Sensor Network Lifetime with Global Energy Balance. IEEE Sens. J. 2015, 15 (9), 5053-5063. 
https://doi.org/10.1109/JSEN.2015.2432114.

7. Chang, J.; Shen, T. An Efficient Tree-Based Power Saving Scheme for Wireless Sensor Networks With Mobile Sink. IEEE Sens. J. 2016, 16 (20), 7545-7557. https://doi.org/10.1109/JSEN.2016.2601327.

8. Liu, X. A Typical Hierarchical Routing Protocols for Wireless Sensor Networks : A Review. IEEE Sens. J. 2015, 15 (10), 5372-5383. https://doi.org/10.1109/JSEN.2015.2445796.

9. Asharioun, H.; Asadollahi, H.; Wan, T. C.; Gharaei, N. A Survey on Analytical Modeling and Mitigation Techniques for the Energy Hole Problem in Corona-Based Wireless Sensor Network. Wireless Personal Communications. 2015, pp 161-187. https://doi.org/10.1007/s11277-014-2122-3.

10. Haseeb, K.; Bakar, K. A.; Ahmed, A.; Darwish, T.; Ahmed, I. WECRR: Weighted Energy-Efficient Clustering with Robust Routing for Wireless Sensor Networks. Wirel. Pers. Commun. 2017, 97 (1), 695-721. https://doi.org/10.1007/s11277-017-4532-5.

11. Jan, N.; Javaid, N.; Javaid, Q.; Alrajeh, N.; Alam, M.; Khan, Z. A.; Niaz, I. A. A Balanced Energy-Consuming and Hole-Alleviating Algorithm for Wireless Sensor Networks. IEEE Access 2017, 5, 6134-6150. https://doi.org/10.1109/ACCESS.2017.2676004.

12. Velmani, R.; Kaarthick, B. An Efficient Cluster-Tree Based Data Collection Scheme for Large Mobile Wireless Sensor Networks. IEEE Sens. J. 2015, 15 (4), 2377-2390. https://doi.org/10.1109/JSEN.2014.2377200.

13. Jung, J. Methods of Cooperative Routing to Optimize The Lifetime of Multi-Hop Wireless Sensor Networks, Ph.D thesis, Georgia Institute of Technology, 2013.

14. Zhu, C.; Wu, S.; Han, G.; Shu, L. E. I. A Tree-Cluster-Based Data-Gathering Algorithm for Industrial WSNs With a Mobile Sink. IEEE Access 2015, 3, 381-396.

15. Salehi_Panahi, M.; Abbaszadeh, M. Proposing a Method to Solve Energy Hole Problem in Wireless Sensor Networks. Alexandria Eng. J. 2017, 12 (5). https://doi.org/10.1016/j.aej.2017.05.012.

16. Jan, N.; Javaid, N.; Javaid, Q.; Alrajeh, N. A.; Alam, M.; Khan, Z. A.; Niaz, I. A. A Balanced Energy Consuming and Hole Alleviating Algorithm for Wireless Sensor Networks. IEEE Access 2016, No. c, 1-1. https://doi.org/10.1109/ACCESS.2017.2676004.

17. Mohemed, R. E.; Saleh, A. I.; Abdelrazzak, M.; Samra, A. S. Energy-Efficient Routing Protocols for Solving Energy Hole Problem In Wireless Sensor Networks. Comput. Networks 2016, 114, 51-66. https://doi.org/10.1016/j.comnet.2016.12.011.

18. Chanak, P.; Banerjee, I.; Rahaman, H. Load Management Scheme for Energy Holes Reduction in Wireless Sensor Networks. Comput. Electr. Eng. 2015, 48, 343-357. https://doi.org/10.1016/j.compeleceng.2015.05.013.

19. Liu, A.; Liu, Z.; Nurudeen, M.; Jin, X.; Chen, Z. An Elaborate Chronological and Spatial Analysis of Energy Hole for Wireless Sensor Networks. Comput. Stand. Interfaces 2013, 35 (1), 132-149. https://doi.org/10.1016/j.csi.2012.06.005.

20. Deng, J. Multi-Hop/Direct Forwarding (MDF) for Static Wireless Sensor Networks. ACM Trans. Sens. Networks 2009, 5 (4), 1-25.

21. Song, C.; Cao, J.; Liu, M.; Zheng, Y.; Gong, H.; Chen, G. Mitigating Energy Holes Based on Transmission Range Adjustment in Wireless Sensor Networks. 5th Int. Conf. Heterog. Netw. Qual. Reliab. Secur. Robustness 2008. https://doi.org/10.4108/ICST.QSHINE2008.3951.

22. Liu, A.; Jin, X.; Cui, G.; Chen, Z. Deployment Guidelines for Achieving Maximum Lifetime and Avoiding Energy Holes in Sensor Network. Inf. Sci. (Ny). 2013, 230, 197-226. https://doi.org/10.1016/j.ins.2012.12.037.

23. Jung, J. W.; Ingram, M. A. Residual-Energy-Activated Cooperative Transmission (REACT) to Avoid the Energy Hole. In 2010 IEEE International Conference on Communications Workshops, ICC 2010; 2010. https://doi.org/10.1109/ICCW.2010.5503966. 
24. Marques, B.; Ricardo, M. Energy-Efficient Node Selection in Application-Driven WSN. Wirel. Networks 2016, 1-30. https://doi.org/10.1007/s11276-016-1194-2.

25. Rasheed, M. B.; Javaid, N.; Khan, Z. A.; Qasim, U.; Ishfaq, M. E-HORM: An Energy-Efficient Hole Removing Mechanism in Wireless Sensor Networks. Can. Conf. Electr. Comput. Eng. 2013. https://doi.org/10.1109/CCECE.2013.6567754.

26. Wang, G.; Wu, K.; Ni, L. M. CSMA/SF: Carrier Sense Multiple Access with Shortest First. IEEE Trans. Wirel. Commun. 2014, 13 (3), 1692-1702. https://doi.org/10.1109/TWC.2014.020414.131457.

27. Sha, C.; Chen, H.; Yao, C.; Liu, Y.; Wang, R. C. A Type of Energy Hole Avoiding Method Based on Synchronization of Nodes in Adjacent Annuluses for Sensor Network. Int. J. Distrib. Sens. Networks 2016, 2016. https://doi.org/10.1155/2016/5828956.

28. Mahani, A.; Kargar, A.; Kavian, Y. S.; Rashvand, H. F. Non-Uniform Distribution of Multi-Hop Sensor Networks: Performance Improvement and Energy Hole Mitigation. IET Wirel. Sens. Syst. 2012, 2 (4), 302308. https://doi.org/10.1049/iet-wss.2012.0032.

29. Chatterjee, P.; Das, N. Coverage Constrained Non-Uniform Node Deployment in Wireless Sensor Networks for Load Balancing. Proc. - Int. Conf. 2014 Appl. Innov. Mob. Comput. AIMoC 2014 2014, 126-132. https://doi.org/10.1109/AIMOC.2014.6785530.

30. Apostolos Demertzis; Oikonomou, K. Avoiding Energy Holes in Wireless Sensor Networks with Nonuniform Node Distribution. IEEE 5th Int. Conf. Information, Intell. Syst. Appl. 2014, 138-143. https://doi.org/10.1109/TPDS.2007.70770.

31. Li, Q. Q.; Gong, H.; Liu, M.; Yang, M.; Zheng, J. On Prolonging Network Lifetime through Load-Similar Node Deployment in Wireless Sensor Networks. Sensors 2011, 11 (4), 3527-3544. https://doi.org/10.3390/s110403527.

32. Khan, M. I.; Gansterer, W. N.; Haring, G. Static vs. Mobile Sink: The Influence of Basic Parameters on Energy Efficiency in Wireless Sensor Networks. Comput. Commun. 2013, 36 (9), 965-978. https://doi.org/10.1016/j.comcom.2012.10.010.

33. Sharma, S.; Jena, S. K. Data Dissemination Protocol for Mobile Sink in Wireless Sensor Networks. J. Comput. Eng. 2014, 2014, 1-10.

34. Wang, J.; Cao, J.; Sherratt, R. S.; Park, J. H. An Improved Ant Colony Optimization-Based Approach with Mobile Sink for Wireless Sensor Networks. J. Supercomput. 2017. https://doi.org/10.1007/s11227-017-2115-6.

35. Nitesh, K.; Kaswan, A.; Jana, P. K. Energy Density Based Mobile Sink Trajectory in Wireless Sensor Networks. Microsyst. Technol. 2017, 1-11. https://doi.org/10.1007/s00542-017-3569-4.

36. Abo-Zahhad, M.; Ahmed, S. M.; Sabor, N.; Sasaki, S. Mobile Sink-Based Adaptive Immune Energy-Efficient Clustering Protocol for Improving the Lifetime and Stability Period of Wireless Sensor Networks. IEEE Sens. J. 2015, 15 (8), 4576-4586. https://doi.org/10.1109/JSEN.2015.2424296.

37. Chen, Y.; Chen, D.; Liu, Y.; Wang, Z. Lifetime Optimization Algorithm with Multiple Mobile Sink Nodes for Wireless Sensor Networks. Springer-Verlag Berlin Heidelb. 2015, 501, 3-13. https://doi.org/10.1007/978-3662-46981-1.

38. Wang, C.; Wu, W. A Load-Balance Routing Algorithm for Multi-Sink Wireless Sensor Networks. IEEE Int. Conf. Commun. Softw. Networks, ICCSN 2009 2009, 380-384. https://doi.org/10.1109/ICCSN.2009.148.

39. Saad, L. Ben; Tourancheau, B.; Saad, L. Ben; Tourancheau, B.; Mobile, M.; Positioning, S.; Networks, S.; Saad, L. Ben; Tourancheau, B. Multiple Mobile Sinks Positioning in Wireless Sensor Networks for Buildings. In SENSORCOMM '09, 3'rd International Conference on Sensor Technologies and Applications, IEEE2009; 2009; pp 264-270. 
40. Zhang, R.; Thiran, P.; Vetterli, M. Virtually Moving Base Stations for Energy Efficiency in Wireless Sensor Networks. 16th ACM Int. Symp. Mob. Ad Hoc Netw. Comput. 2015, 357-366. https://doi.org/10.1145/2746285.2746291.

41. Gomez-Pulido, J. A.; Lanza-Gutierrez, J. M. Reliability and Efficiency in Wireless Sensor Networks: Heuristic Approaches. J. Heuristics 2015, 21 (2), 141-143. https://doi.org/10.1007/s10732-014-9263-0.

42. Mahajan, S.; Malhotra, J.; Sharma, S. Energy Balanced Heuristic Approach for Path Selection Using Graph Theory. Procedia Comput. Sci. 2015, 46 (2015), 101-108. https://doi.org/10.1016/j.procs.2015.01.063.

43. Samara, G.; Aljaidi, M. Aware-Routing Protocol Using Best First Search Algorithm in Wireless Sensor. Int. Arab J. Inf. Technol. 2018, 15 (3), 592-598.

44. Li, X. H.; Hong, S. H.; Fang, K. L. WSNHA-GAHR: A Greedy and A* Heuristic Routing Algorithm for Wireless Sensor Networks in Home Automation. IET Commun. 2011, 5 (13), 1797-1805. https://doi.org/10.1049/iet-com.2010.0746.

45. Moghadam, M. N.; Taheri, H. High Throughput Load Balanced Multipath Routing in Homogeneous Wireless Sensor Networks. IEEE 22nd Iran. Conf. Electr. Eng. ICEE 2014 2014, No. Icee, 1516-1521. https://doi.org/10.1109/IranianCEE.2014.6999775.

46. Biswas, K.; Muthukkumarasamy, V.; Sithirasenan, E.; Singh, K. An Energy Aware Heuristic-Based Routing Protocol in Wireless Sensor Networks. IEEE17th Int. Conf. Comput. Inf. Technol. 2014, 53-58.

47. Hwang, S.-F.; Lin, H.-H.; Dow, C.-R. An Energy-Efficient Routing Protocol in Wireless Sensor Networks with Holes. IEEE Proc. 4th ICUFN 2012, 17-22.

48. Li, S.; Gao, H.; Wu, D. An Energy-Balanced Routing Protocol with Greedy Forwarding for WSNs in Cropland. IEEE Int. Conf. Electron. Inf. Commun. Technol. 2016, 1-7.

49. Medjiah, S.; Ahmed, T.; Krief, F. AGEM : Adaptive Greedy-Compass Energy-Aware Multipath Routing Protocol for WMSNs. IEEE 17th Consum. Commun. asd Netw. Conf. 2010, 1-6.

50. Kamal, A. R. M.; Hamid, M. A. Supervisory Routing Control for Dynamic Load Balancing in Low Data Rate Wireless Sensor Networks. Wirel. Networks 2016, 23 (4), 1085-1099. https://doi.org/10.1007/s11276-0161209-z.

51. Jia, W.; Wang, T.; Wang, G.; Guo, M. Hole Avoiding in Advance Routing in Wireless Sensor Networks. IEEE Proc WCNC 2007.

52. Pathak, A.; Zaheeruddin; Lobiyal, D. K. Maximization the Lifetime of Wireless Sensor Network by Minimizing Energy Hole Problem with Exponential Node Distribution and Hybrid Routing. IEEE Students Conf. Eng. Syst. SCES 2012 2012. https://doi.org/10.1109/SCES.2012.6199106.

53. Choi, S. Y.; Kim, J. S.; Han, S. J.; Choi, J. H.; Rim, K. W.; Lee, J. H. Dynamic Routing for Mitigating the Energy Hole Based on Heuristic Mobile Sink in Wireless Sensor Networks. In International conference on Advances in computer science and information technology,; 2010; pp 159-174. https://doi.org/10.1007/978-3-64213577-4_14.

54. Chen, J.; Jia, J.; Wen, Y.; Zhao, D. Towards an Optimal Energy Consumption for Unattended Mobile Sensor Networks through Autonomous Sensor Redeployment. Sci. World J. 2014, 2014 (April). https://doi.org/10.1155/2014/716838.

55. Ahadi, M.; Bidgoli, A. M. A Multiple-Sink Model for Decreasing the Energy Hole Problem in Large-Scale Wireless Sensor Networks. Int. J. Comput. Theory Eng. 2012, 4 (5), 843-846.

56. Mittal, N.; Singh, U.; Sohi, B. S. A Stable Energy Efficient Clustering Protocol for Wireless Sensor Networks. Wirel. Networks 2016, March (6), 1809-1821. https://doi.org/10.1007/s11276-016-1255-6. 
57. Shebli, F.; Dayoub, I.; M'foubat, a. O.; Rivenq, a.; Rouvaen, J. M. Minimizing Energy Consumption within Wireless Sensors Networks Using Optimal Transmission Range between Nodes. 2007 IEEE Int. Conf. Signal Process. Commun. 2007, 105-108. https://doi.org/10.1109/ICSPC.2007.4728266.

58. Imon, S. K. A.; Khan, A.; Di Francesco, M.; Das, S. K. Energy-Efficient Randomized Switching for Maximizing Lifetime in Tree-Based Wireless Sensor Networks. IEEE/ACM Trans. Netw. 2015, 23 (5), 14011415. https://doi.org/10.1109/INFCOM.2013.6567102. 


\section{Author Contribution:}

Conceptualization: MSA Mustafa, BM Ali, MDA Rasid, SJB Hashim

Methods: MSA Mustafa

Software: MSA Mustafa

Validation: BM Ali, MDA Rasid, SJB Hashim

Formal Analysis: MSA Mustafa, WAW Adnan

Investigation: MSA Mustafa

Resources: BM Ali

Data curation: MSA Mustafa, BM Ali, WAW Adnan

Visualization: MSA Mustafa

Supervision: BM Ali, MDA Rasid, SJB Hashim

Project administration: MSA Mustafa, BM Ali 